\title{
Comparison of several biological indices based on river macroinver- tebrate benthic community for assessment of running water quality
}

E. Ricol

A. Rallol

M.A. Sevillano ${ }^{1}$

M.L. Arretxe ${ }^{1}$

Keywords : Biological indices, water quality, macroinvertebrates, rivers.

Nine biological indices based on the macroinvertebrate community inhabiting rivers have been calculated in order to find out which of them is more appropriate to evaluate the qualitative status of the running water for public environmental authorities. The BMWP' score (Alba-Tercedor \& Sánchez-Ortega 1988) has been chosen because it is both accurate and precise, and easy to calculate. Some problems derived from sampling strategies are discussed.

Comparaison de plusieurs indices biologiques basés sur la communauté macroinvertébrée benthique des rivières pour évaluer la qualité des eaux courantes

Mots clés: Indices biotiques, qualité de l'eau, macroinvertébrés, fleuves.

Neuf indices biologiques d'évaluation de la qualité des eaux fluviales basés sur l'étude des macroinvertébrés benthiques sont comparés. Par sa simplicité et sa précision, l'indice BMWP' (Alba-Tercedor \& Sánchez-Ortega 1988) paraît être l'indice le plus pratiçue pour être employé par les organismes publics de gestion des eaux. Quelques problèmes relatifs aux valeurs obtenues par les indices à cause des stratégies d'échantillonnage suivies sont commentés.

\section{Introduction}

The saprobien-system (Kolkwitz \& Marsson 1902, 1908,1909 ) was the first proposed way to evaluate the quality and status of fresh waters by biological methods. It has been revised and updated several times since, and adapted to different taxocenosis (Liebmann 1951, 1962 ; Sladecek 1961, 1967, 1973 ; Fjerdingstad 1964) and specially used in central and eastern Europe.

Also, there are other methods based on the study of the faunistic community, such as the assessment of taxonomic richness and diversity or trophic structure. Most popular in West Europe are the Biotic indices that rely on the presence of diverse taxons chosen for their specific sensitivity towards pollu-

1. Universidad del País Vasco. Facultad de Ciencias. Departamento de Biologia Animal y Gencetica, Zoologia. Apdo. 644 E-48080 Bilbao, España. tion. Several indices of this kind have been proposed, each of them widely applied to most rivers in any specific geographical area. So, in Great Britain the TBI, the CBS and the EBI indices (see ahead for definition) are the most commonly used, whereas in France the VT and IBG are generally preferred. Actually it seems very interesting to compare their different results when applied to the same biological entity (Balloch et al. 1976 ; Ghetti \& Bonazzi 1977 ; Tolkamp 1985 ; Mesanza et al. 1988), specially in order to choose one of them and adjust it to a particular fluvial system.

The major part of the fluvial system of the Basque Country (northern Spain) is seriously polluted and spoiled. In order to evaluate this situation, several studies - supported by the Administration -, have been carried out to get a general map of river water quality and to propose a management strategy. A preliminary problem is choosing a suitable biotic index, both accurate and workable. A theoretical method of comparison between indices has 
been designed and tested with data from basque rivers, both in analytical and holistic ways.

\section{Methods}

The biotic indices calculated and compared are :

TBI : Trent Biotic Index (Woodiwiss 1964)

EBI : Extended Biotic Index (Woodiwiss 1978)

VT : Indice Biotique (Verneaux \& Tuffery 1967)

IBG : Indice Biologique de Qualité Générale (Verneaux et al. 1982)

BMWP' : Biological Monitoring Working Party, adapted to Iberian Peninsula (Alba-Tercedor \& Sánchez-Ortega 1988)

ASPT : Average Score per Taxon (Armitage et al. 1988) derived from BMWP'

CBS : Chandler's Biotic Score (Chandler 1970)

ACBS : Average Chandler's Biotic Score (Balloch et al. 1976)

RVI : River of Vaud Index (Lang et al. 1989).

The comparison between them is made by theoretical considerations (see results), and by Pearson product-moment correlation analysis (Sokal \& Rohlf 1969). The scores are categorised by hierarchic clustering analysis, evaluating square euclidean distances and grouping the results by the UPGMA algorithm (Sneath \& Sokal 1973).

The data were obtained from 65 fluvial stations in Alava and Guipúzcoa (Basque Country, Spain (Fig. 1), whose rivers are short, shallow and turbulent and with a fluctuating streamflow upon a predominantly calcareous lithology. Samples were taken by a " kicker " handnet $(500 \mu \mathrm{m} \varnothing)$ in lotic system, looking for the biggest spatial heterogeneity ; over whole, a minimum of $4500 \mathrm{~cm}^{2}$ area were sampled each time. Faunistic analysis included Annelida, Mollusca, Crustacea, Ephemeroptera, Plecoptera, Odonata, Heteroptera, Coleoptera, Megaloptera, Trichoptera and Diptera.

\section{Results and discussion}

When choosing a biological method to evaluate the quality of running water, two considerations must be borne in mind (Verneaux 1982) : firstly, the accuracy and precision, that is to say, the adjustment of the obtained results to the real fluvial condition that is being studied, and secondly, the practicality and simplicity in the use and application of the method.
The exigencies of these two methodological aspects are, in some way, contradictory : accuracy requires a carefully, time-consuming and detailed identification up to the species level, because species is the unquestionable ecological unity (unambiguously linked to the ecological niche concept on which bioindication takes root). It is evident that this exigence is neither simple nor practical, and practicality and simplicity are required because of the large number of samples which have to be processed as fast as possible and with a minimum of taxonomic analysis dedication. So we have to choose the method that, being simple and practical enough, shows the highest correlation with the most accurate index which is that which uses the species level as the taxonomic unity.

The biotic indices applied in our work could be classified in three groups, according to the taxonomical level required. The simplest ones, IBG, BMWP' and ASPT, only require the taxonomic family level to be worked out. More exigent are TBI, EBI, VT and RVI, that need identification up to families and genera, and the most difficult are those that require identification at the genera and species level, as CBS and its derivated ACBS do, so that only trained taxonomists can do the work. But when looking for precision and accuracy the CBS index must be preferred, as not only the theoretical considerations we have already made but also practical results have shown (Balloch et al. 1976, Washington 1984, Domezain et al. 1987, Mesanza et al. 1988).

All the above mentioned indices have been applied to the data from the rivers of Alava and Guipúzcoa : the results are shown in Table II, that also include their taxons' number and the values of Shannon-Weaver faunistic diversity index in each sample. Comparisons have been established between all these values which have been referred to CBS index (Fig. 2) that has been chosen because of its aforementioned accuracy in order to evaluate the behaviour of the other more practical indices. The interrelation between the indices is also studied by Pearson linear correlation analysis (Table 1).

CBS index has been shown to be very sensitive to small variations in water quality. The range of the obtained values is wider for each value of the other indices, specially the highest TBI, EBI and VT values. 


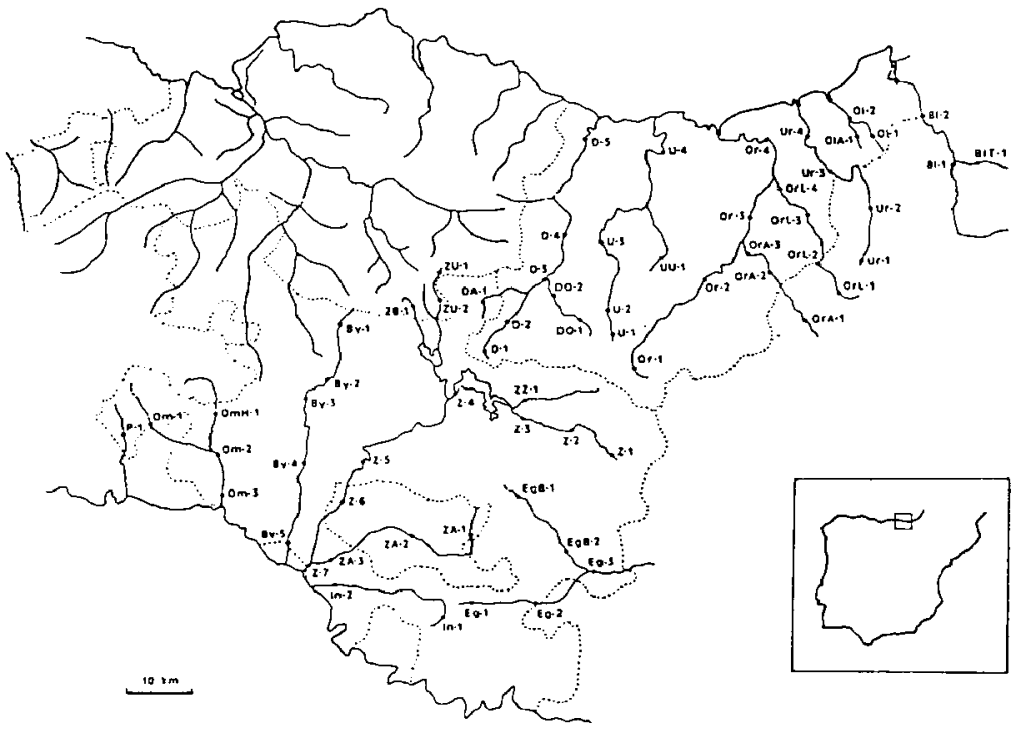

\begin{tabular}{|c|c|c|c|c|}
\hline Sites & Piver & Alnitude & $\begin{array}{l}\text { Distance from } \\
\text { sourcd (Km) }\end{array}$ & Locality \\
\hline 01 & Donom & 460 & 3.5 & Salinas de Laniz \\
\hline D2 & Deos & 280 & 10 & Estronaria \\
\hline 03 & Deos & 270 & 20.5 & San Prodencio \\
\hline 04 & Dobs & 120 & 30.5 & Makoialde \\
\hline DS & Doba & 20 & 50.5 & Puente Sasola \\
\hline DA1 & Aramuian & 340 & 3 & Doarfa \\
\hline DO1 & Orate & 275 & 1.5 & Oaburriera \\
\hline$\infty$ & OAalo & 210 & $\theta$ & Zubillaga \\
\hline U1 & Whole & 580 & $t .5$ & Irumugarrieta \\
\hline ty2 & Urole & 420 & 6.5 & Mirandaola \\
\hline U3 & Urol: & 210 & 20.5 & Antzpurutroso \\
\hline 44 & Unola & 20 & 46.5 & Arracnazabal \\
\hline UU1 & Uetestrila & 240 & 2.5 & Arsiz Madainenta \\
\hline Or 1 & Oris & 620 & 3 & Zegama \\
\hline 0.2 & Oria & 100 & 26 & Legorata \\
\hline $\mathrm{Cr} 3$ & Ona & 80 & 41 & irurat Anoula \\
\hline$O_{r}$ & ona & 15 & 57 & Zubiet \\
\hline Ort-1 & Leizaran & 540 & 5.5 & Lenta \\
\hline Orl2 & Lemzeran & 40 & 11.5 & Loitza \\
\hline Ort3 & Lemeren & 240 & 25.5 & Luizatan \\
\hline Ort & Leizaran & 70 & 35 & Andouin \\
\hline Orat & Araxes & 260 & 3 & Belah \\
\hline OA2 & Araxes & 140 & 13 & Liatza \\
\hline Oras & Araxes & 100 & 19 & Truams \\
\hline U:t & Urumea & 550 & 2.5 & Lentadarrea \\
\hline Ur2 & Unumea & 140 & 145 & Goizuel: \\
\hline U.3 & Urumas & 40 & 29.5 & Ugaldeoxo \\
\hline Wis & Unuma & 5 & 41.5 & Eroobia \\
\hline Oi1 & Oyarzun & 100 & 5 & Aibulage \\
\hline 0.2 & Oyarzun & 40 & 11 & Ugaldetio \\
\hline OAl 1 & miluber & 70 & 5 & Kanka \\
\hline Bi? & Gudesoz & 50 & 42.5 & Voma Yand \\
\hline Bu2 & Buteson & 5 & 555 & Endarlatra \\
\hline
\end{tabular}

\begin{tabular}{|c|c|c|c|c|}
\hline Sires & Piver & nilude & $\begin{array}{l}\text { Distance hom } \\
\text { sounce (Km) }\end{array}$ & Loentity \\
\hline $\mathrm{BRT}$ & Txumistra & 100 & 5 & Exalaz \\
\hline$P_{\dagger}$ & Purion & 750 & 7.5 & Alowera \\
\hline Om1 & Orecillo & & B & Korro \\
\hline $\operatorname{am2}$ & Omecillo & 520 & 28 & Venta Blanca \\
\hline $0 \mathrm{~m} 3$ & Omecilla & 490 & 38 & Berguienda \\
\hline Onnt1 & Humecillo & 570 & 9 & Osma \\
\hline By" & Bayas & 700 & 45 & Sarria \\
\hline By2 & Bayas & 590 & 16.5 & Lukiano \\
\hline By3 & Eayas & 580 & 23.5 & Katadiano \\
\hline Bys & Bayas & 520 & 36.5 & Subiama frotes \\
\hline Bys & Bayss & 475 & 53.5 & Plovolthasa \\
\hline 21 & Zadorra & 040 & 0.5 & Munain \\
\hline 22 & Zadorra & 580 & 5.5 & Salvanesra \\
\hline 23 & Zadorra & 500 & 15 & Eivra \\
\hline $\mathbf{Z 4}$ & Zadorra & 520 & 30 & Asroiaco \\
\hline 25 & Zadorra & 500 & 52 & Villodas \\
\hline $\mathbf{2 8}$ & Zacorra & 480 & 6 & Puebla do Arganzon \\
\hline 27 & Zadoera & 450 & $\pi$ & Arte \\
\hline $2 \mathrm{N1}$ & Uitkiola & 650 & 0.2 & Unkrolete \\
\hline ZUR & Urkioia & 560 & 6 & Otonndio \\
\hline 281 & Zuoizabala & 580 & 3.5 & Uobidean. \\
\hline Z्रा & Barundia & 560 & 15 & Ozata \\
\hline Za1 & Anoda & 650 & 6 & Sasatz: \\
\hline 242 & Anda & 540 & 20.5 & Vonta Armentia \\
\hline Zns & Ayuda & 470 & 36 & Esangan: \\
\hline In: & Inglares & 840 & 2.5 & Plosón \\
\hline $\ln 2$ & Inglertes & 505 & 23.5 & $\infty$ \\
\hline Eg1 & Et & 708 & 2.2 & Lagrin \\
\hline Eg2 & $\mathrm{Eg}$ & 600 & 13.4 & Angoscina \\
\hline$E \theta$ & Exa & 550 & 27,8 & Sla Curz de Camoezo \\
\hline Eog1 & Berrón & 820 & 1.5 & Aracela \\
\hline Ege? & Bemon & 600 & 15.5 & Antorana \\
\hline
\end{tabular}

Fig. 1. Localisation et caractéristiqus des points d"échantillonnage.

Fig. 2. Location and characteristics of sampling sites in the study area. 

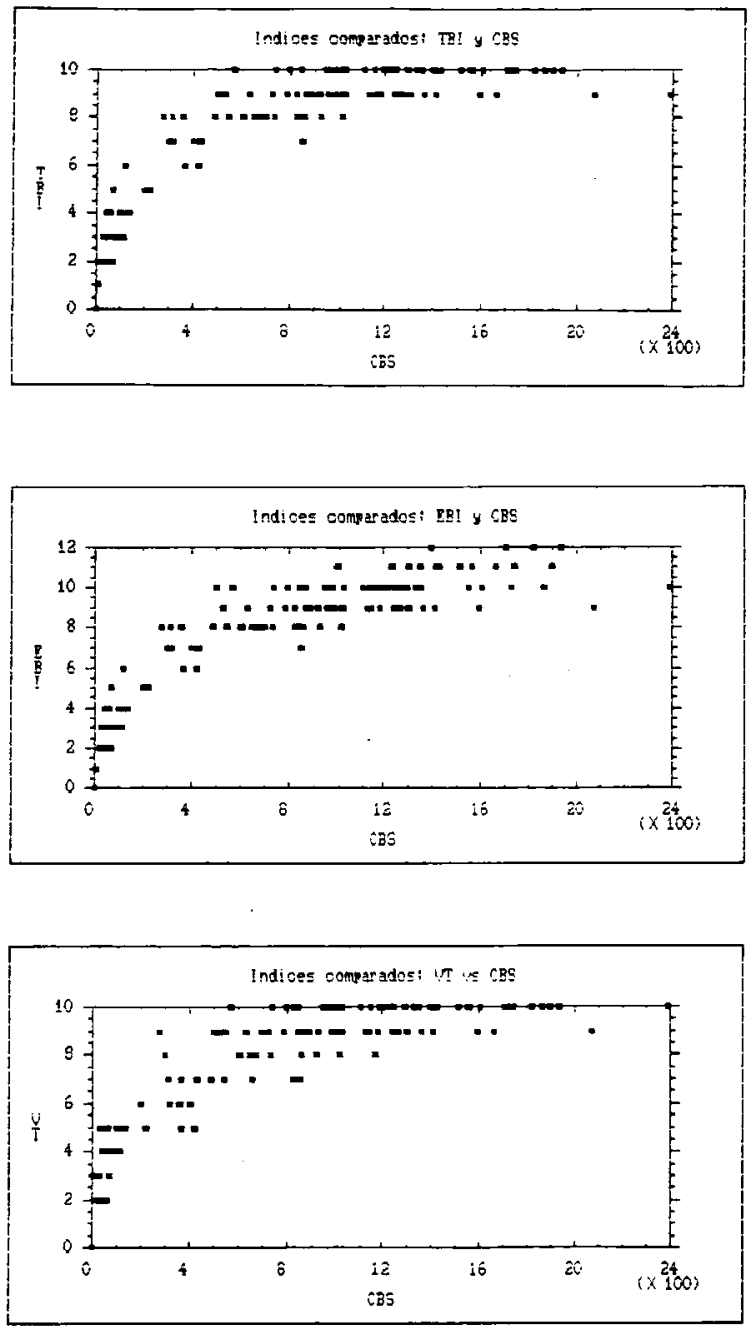

Fig. 2. Représentation des valeurs des différenıs indices biologiques en fonction des celles du CBS. Fig. 2. Relationship between the CBS and the other biological indices. 

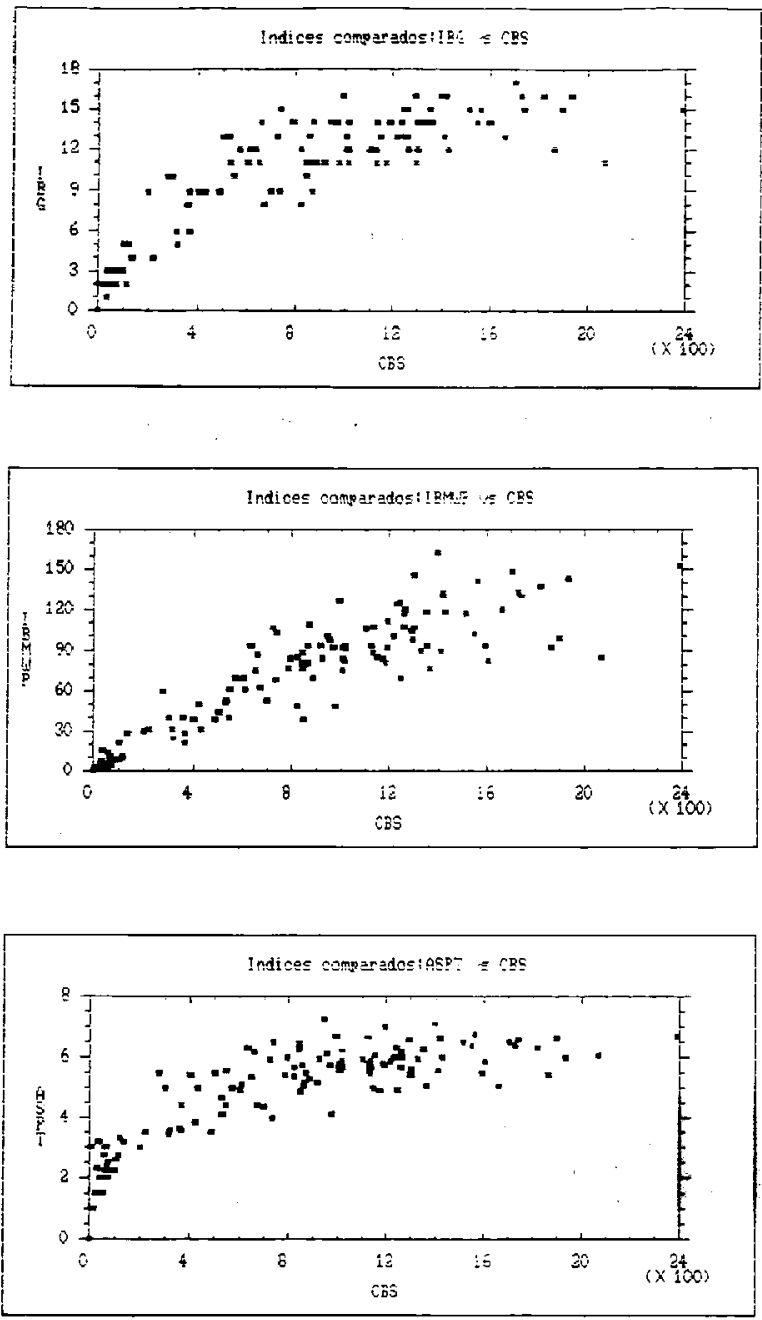

Fig. 2. Representation des valeurs des différents indices biologiques en fonction des celles du CBS.

Fig. 2. Relationship between the CBS and the other biological indices. 

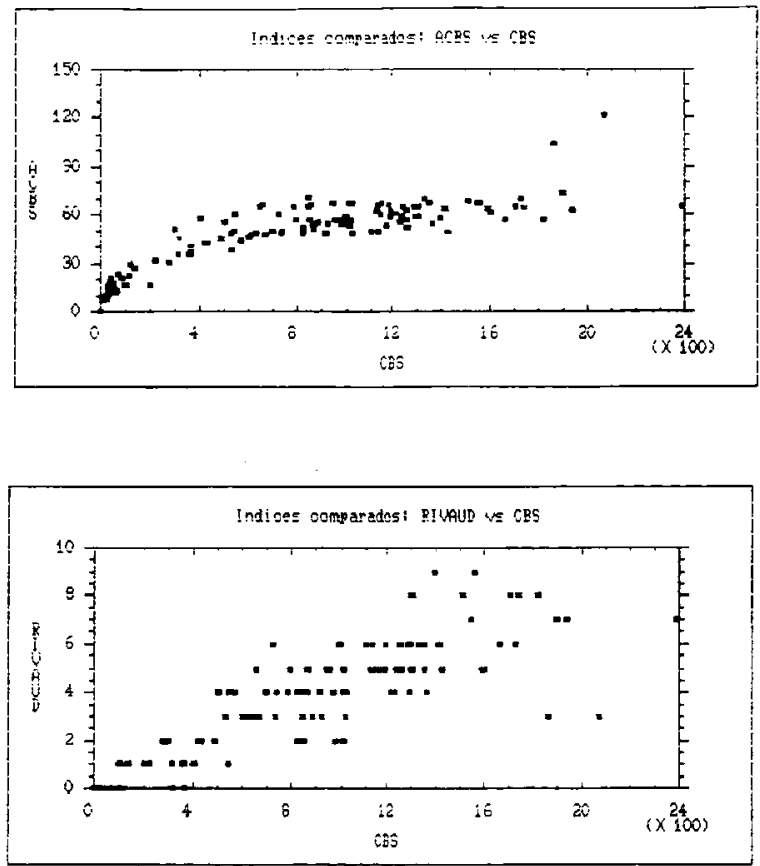

Fig. 2. Représentation des valeurs des différents indices biologiques en fonction des celles du CBS. Fig. 2. Relationship between the CBS and the other biological indices.

Tableau 1. Valeurs des coefficients de corrélation linéaire entre les différents indices biologiquues $(\mathrm{n}=127$ ).

Table 1. Linear correlation values between the biological indices $(\mathbf{n}=127$ ).

\begin{tabular}{lccccccccc}
\hline & TBI & EBI & VT & IEG & BMWP & ASPT & CBS & ACBS & RVI \\
\hline TBI & 1.000 & & & & & & & & \\
EBI & .9865 & 1.000 & & & & & & & \\
VT & .9668 & .9542 & 1.000 & & & & & & \\
IBG & .9378 & .9453 & .9335 & 1.000 & & & & & \\
BMWP' & .8608 & .8984 & .8665 & .9138 & 1.000 & & & & \\
ASPT & .9240 & .9174 & .9404 & .9317 & .8897 & 1.000 & & & \\
CBS & .8174 & .8475 & .8162 & .8523 & .8959 & .8105 & 1.000 & & \\
ACBS & .8751 & .8574 & .8606 & .8560 & .8880 & .8779 & .8510 & 1.000 & \\
RVI & .8317 & .8744 & .8326 & .8956 & .9405 & .8413 & .8743 & .7568 & 1.000 \\
\hline
\end{tabular}


These three indices have a very similar design, and consequently show a high degree of linear correlation between them $(r>0.95$, g.f. $=125 ; p \leqslant 0.001$ - Table 1 -) and the lowest with CBS. The great variability in the highest values reached by the three indices makes the relation to CBS to fit more to a logarithmic model than a linear one. So, in our case, they work well in polluted waters but fail to evaluate adequately the quality of good water. It is important to notice that VT is, so far, the most commonly used index in previous studies in spanish rivers, so that its calculation would be interesting for a short period in order to compare with historical situations.

The highest linear correlation with CBS is reached by BMWP' ( $r=0.8959)$, RVI ( $r=0.8743)$ and IBG $(r=0.8523)$ indices (in all cases, with g.f. $=125$ and $\mathrm{p} \leqslant 0.001$, Table 1 ). The RVI is a very recently proposed index (Lang 1989) to evaluate the quality of rivers in the Vaud canton, Switzerland, and is based upon the total number of taxons in a sample and the number of them which are intolerant to pollution. The range of variation of CBS against RVI is very wide in all cases, even those corresponding to waters of the lowest quality. So, RVI is not able to discern, in our country, small variations in water quality.

IBG (Verneaux et al. 1982) seems to be more sensible and accurate than its antecesor VT, and by its application a really good, extensive and complete characterization of the fluvial section is achieved. Nevertheless, its estimation requires a well-trained and skilled staff and demands a very rigourous and complex sampling protocol which is difficult to follow when a large fluvial net must be studied against time. The sampling method in the present work did not fit that protocol, so the values of the index obtained must be accepted only as approximations. Also, the laboriousness and thoroughness of the method is far from being simple, so this index is not suitable for our purpose.

The BMWP' is an index derived from the british BMWP (National Water Council 1981), adapted to the faunistic peculiarities of the iberian peninsula by Alba-Tercedor \& Sánchez-Ortega (1988). It is able to detect small variations in water-quality (see Fig. 2), and shows a very high linear correlation with CBS ( $r=0.8959$; d.f. $=125 ; p \leqslant 0.001$ ). It is one of the simplest indices to calculate, since it requires a taxonomic analysis only down to the family level. The other index, ASPT, is a modification of the BMWP (in the same way that ACBS is of the CBS) to correct the values obtained from a very particular fluvial conditions : when the faunistic scarcity is not an effect of pollution but of the small biogenic capacity of the ecosystem (as it actually happens near the river sources). So, these indices are contempled only as auxiliaris to be applied only when such conditions are present.

The BMWP' and the IBG have already been applied to a few basque rivers (Lea, Oria and Bidasoa) by Rodriguez \& Wright (1988), who obtained values remarkably higher than ours (in Oria and Bidasoa rivers). As the biological condition of both rivers have not change, this effect can be attributed to the mean of sampling, as these authors took fauna not oniy from lotic but from lentic and marginal zones, also. It is obviously known that lentic and marginal sampling raises the number of taxons, as also has been proved by Rodriguez \& Wright (1991) ; Mesanza et al. (1988) have got results similar to ours in the Lea river.

The normalised data in Table 2 (indices, number of taxons and diversity values for each sample) have been analysed by clustering methods. We have found six well defined groups of sampling stations that correspond to six correlative ranges of BMWP' values (Table 3), substantially similar to those determined by Alba-Tercedor \& Sánchez-Ortega (1988) according to Ghetti et al. (1983). To classify the results of Rodriguez \& Wright (1988) in these categories would give way to an overvaluation of the real quality of the water. This is the outcome of the methodological discrepancies again : Alba-Tercedor \& Sánchez-Ortega (1988) included lotic and lentic zones in their sampling strategy ant the concordance with our results may be a consequence of the nature of their faunistic data, from Sierra Nevada (Andalucía) rivers, poorer than ours (we have personally discussed all these items with Dr. Alba-Tercedor).

Other iberian rivers looked more like Basque than Andalucian ones : the BMWP' range values are very similar to ours in the Tietar river (western Spain) (Garcia-Avilés, pers. comm.), in the Júcar basin (eastern Spain) and in Gredos Mountains (central Spain) fluvial systems (Dr González, in litt.). So we wonder if a unique way of application of the BMWP' for all the iberian peninsula is allowed. 
Tableau 2. Valeurs des indices de qualité des eaux, de la diversité de Shannon-Weaver (H) et nombre de taxons (N). Sp : Printemps, Au : Automne.

Table. 2. Values of water quality indices, diversity index of Shannon-Weaver $(\mathrm{H})$ and number of taxa (N). Sp : Spring, Au : Autumn.

\begin{tabular}{|c|c|c|c|c|c|c|c|c|c|c|c|c|c|c|c|c|c|c|c|c|c|c|}
\hline \multirow{3}{*}{$\overline{\mathrm{D} 1}$} & \multirow{2}{*}{\multicolumn{2}{|c|}{$\begin{array}{c}\mathrm{TBI} \\
\text { sp } \mathrm{Au}\end{array}$}} & \multicolumn{2}{|r|}{ EBI } & \multicolumn{2}{|r|}{$\mathrm{VT}$} & & IBS & & MWP" & & ASPT & & CBS & & CBs & & RVI & & $\mathrm{H}$ & & $\mathbf{N}$ \\
\hline & & & & SP AL & & p Au & & & $s$ & $P \quad A U$ & $S p$ & Au & sp & $\mathrm{Au}$ & $\mathrm{sp}$ & Au & & $\mathrm{p} A \mathrm{u}$ & $\mathrm{sp}$ & $\mathrm{AL}$ & & $\mathrm{P} \mathrm{Au}$ \\
\hline & 10 & 9 & 10 & 9 & 10 & 9 & 14 & 13 & 101 & 51 & 7.21 & 4.64 & 948 & 529 & 67.74 & 48.09 & 5 & 3 & 3.03 & 2,49 & 26 & 18 \\
\hline $\mathrm{D} 2$ & 2 & 2 & 2 & 2 & 2 & 2 & 2 & 2 & 3 & 3 & 1.50 & 1.50 & 54 & 30 & 18.00 & 15.00 & 0 & 0 & 0.46 & 1.41 & 6 & 8 \\
\hline D3 & 2 & 2 & 2 & 2 & 2 & 2 & 2 & 2 & 1 & 3 & 1.00 & 1.50 & 19 & 31 & 9.50 & 10.33 & 0 & 0 & 1.36 & 1.47 & 4 & 6 \\
\hline 04 & 2 & 3 & 2 & 3 & 2 & 4 & 2 & 3 & 3 & 9 & 1.50 & 2.25 & 38 & 99 & 19.00 & 16.50 & 0 & 0 & 2.24 & 2.01 & 7 & 9 \\
\hline DS & 3 & 2 & 3 & 2 & 3 & 2 & 2 & 2 & 7 & 3 & 2.33 & 1.50 & 32 & 26 & 10.60 & B. 66 & 0 & 0 & 2.60 & 1,44 & 15 & 7 \\
\hline DA1 & 9 & 9 & 9 & 9 & 9 & 10 & 14 & 11 & 93 & 82 & 6.20 & 5.86 & 1019 & 1016 & 67.90 & 56.44 & 4 & 3 & 3.15 & 0.92 & 29 & 22 \\
\hline Do1 & 10 & 8 & 10 & 8 & 10 & 9 & 14 & 11 & 89 & 80 & 5.93 & 6.28 & 1327 & 847 & 69.84 & 65.15 & 6 & 3 & 2.94 & 2.05 & 29 & 19 \\
\hline$D 02$ & 6 & 3 & 6 & 3 & 5 & 4 & 5 & 3 & 10 & 6 & 3.33 & 2.00 & 119 & 43 & 29.75 & 21,50 & 0 & 0 & 2.62 & 1.84 & 日 & 11 \\
\hline U1 & 10 & 10 & 11 & 10 & 10 & 10 & 16 & 14 & 127 & 102 & 6.68 & 6.37 & 996 & 1546 & 58.58 & 67.21 & 6 & 7 & 4.01 & 3.94 & 31 & 28 \\
\hline v2 & 9 & 8 & 9 & 8 & 9 & 9 & 11 & 10 & 85 & 61 & 6.07 & 5.54 & 2070 & 547 & 121.76 & 60.77 & 3 & 4 & 3.41 & 3.81 & 32 & 21 \\
\hline U3 & 5 & 3 & 5 & 3 & 4 & 5 & 3 & 3 & 5 & 16 & 2.50 & 3.20 & 72 & 36 & 23,60 & $12+00$ & 0 & 0 & 2.27 & $2+59$ & 7 & 13 \\
\hline Ua & 9 & 7 & 9 & 7 & 9 & 6 & 11 & 5 & 53 & 25 & 4.07 & 3.57 & 532 & 319 & 38.00 & 45.57 & 3 & 0 & 2.41 & 2.23 & 24 & 13 \\
\hline UU1 & 10 & 9 & 12 & 10 & 10 & 10 & 16 & 15 & 143 & 153 & 5.96 & 6.65 & 1935 & 2390 & 62.41 & 64.59 & 7 & 7 & 3.77 & 4.70 & 47 & 45 \\
\hline Or1 & 10 & 10 & 10 & 11 & 10 & 10 & 16 & 15 & 233 & 131 & 6.33 & 6.55 & 1727 & 1740 & 70.08 & 64,44 & 6 & 8 & 3.22 & 3.89 & 29 & 39 \\
\hline Or2 & B & 8 & 8 & $B$ & 7 & 7 & 10 & 6 & 40 & 31 & 4.44 & 3.44 & 543 & 316 & 49.36 & 35.11 & 1 & 1 & 1.95 & 3.58 & 19 & 22 \\
\hline or3 & 4 & 3 & 4 & 3 & 2 & 5 & 2 & 3 & 6 & 12 & 2.00 & 2.40 & 46 & 67 & 12.50 & 13.40 & 0 & 0 & 2.07 & 0.86 & 15 & 8 \\
\hline or 4 & 3 & 4 & 3 & 4 & 2 & 5 & 1 & 5 & 3 & 21 & 1.50 & 2.62 & 34 & 103 & 11.33 & 17.16 & 0 & 1 & 2.24 & 3.10 & 20 & 18 \\
\hline orti & 10 & 10 & 12 & 11 & 10 & 10 & 27 & 15 & 149 & 141 & 6.48 & 6.71 & 1704 & 1560 & 65.50 & 67.82 & 8 & 9 & 4.36 & 3.61 & 40 & 36 \\
\hline orL2 & 9 & 9 & 10 & 9 & 8 & 10 & 11 & 13 & 83 & 92 & 4.88 & 5.75 & 1171 & 1010 & 53.22 & 53.15 & 5 & 5 & 2.33 & 1.26 & 36 & 22 \\
\hline arL3 & 10 & 9 & 10 & 9 & 10 & 9 & 13 & 12 & 69 & 94 & 4.93 & 6.27 & 1245 & 632 & 65.52 & 48.61 & 5 & 3 & 3.30 & 2.35 & 29 & 20 \\
\hline OrL 4 & 9 & 10 & 9 & 10 & 9 & 10 & 13 & 14 & 108 & 92 & 5.68 & 5.75 & 1257 & 972 & 57.13 & 57.17 & 6 & 4 & 3.42 & 4.07 & 41 & 35 \\
\hline OrA1 & 9 & 7 & 9 & 7 & 9 & 8 & 12 & 10 & 88 & 40 & 5.50 & 5.00 & 1132 & 302 & 62.88 & 50.33 & 6 & 2 & 2.87 & 1.97 & 21 & 11 \\
\hline OrA2 & 9 & $\theta$ & 9 & $B$ & 9 & 9 & 13 & 11 & 85 & 83 & 5.00 & 5.93 & 1146 & 927 & 60.31 & 54.52 & 5 & 3 & 3.54 & 2.55 & 22 & 21 \\
\hline OrA3 & 9 & 9 & 30 & 9 & 9 & 9 & 11 & 9 & ล 1 & 93 & 5.08 & 5.17 & 867 & 868 & 54.18 & 51.05 & 5 & 4 & 3.42 & 3.76 & 27 & 26 \\
\hline $\mathrm{Ur} 1$ & 10 & 10 & 11 & 10 & 10 & 10 & 16 & 13 & 132 & 85 & 6.60 & 6.07 & 1414 & 1151 & 64.27 & $6 ? .70$ & 6 & 5 & 3.70 & 2.96 & 33 & 21 \\
\hline Ur2 & 9 & 10 & 9 & 10 & 9 & 10 & 14 & 14 & 76 & 212 & 5.43 & 7.00 & 787 & 1193 & 65.58 & 62.78 & 4 & 6 & 2.04 & 2.62 & 18 & 23 \\
\hline Ur3 & 9 & 9 & 10 & 9 & 9 & 10 & 15 & 14 & 117 & 98 & 6.16 & 6.12 & 1259 & 956 & 62.95 & 56.23 & 6 & 5 & 3.78 & 2.69 & 35 & 24 \\
\hline Ur4 & 3 & 3 & 3 & 3 & 5 & 4 & 2 & 3 & 14 & 9 & 2.80 & 2.25 & 59 & 84 & 14.75 & 21.00 & 0 & 0 & 2.73 & 0.96 & 13 & 9 \\
\hline Oi1 & 9 & 10 & 9 & 10 & 9 & 10 & 15 & 14 & 126 & 84 & 6.30 & 6.00 & 1240 & 797 & 59.04 & 56.92 & 5 & 5 & 3.60 & 1.86 & 32 & 20 \\
\hline 012 & 7 & 2 & 7 & 2 & 6 & 4 & 9 & 3 & 38 & 12 & 5.43 & 3.00 & 403 & 64 & 57.57 & $12, \mathrm{BO}$ & 1 & 0 & 2.91 & 2.80 & 19 & 12 \\
\hline OIAI & $\theta$ & 9 & 8 & 9 & 7 & 9 & 34 & 13 & 86 & 75 & 6.14 & 5.77 & 659 & 1007 & 65.90 & 67.13 & 5 & 4 & 2.47 & 2.20 & 20 & 20 \\
\hline Bil & 10 & 10 & 10 & 11 & 10 & 10 & 12 & 12 & 92 & 119 & $5: 41$ & 5.95 & 1864 & 1425 & 103.50 & 49.13 & 3 & 5 & 3.29 & 3.86 & 27 & 38 \\
\hline Bi 2 & 9 & 8 & 10 & $B$ & 9 & B & 23 & 11 & 44 & 69 & 5.50 & 4.93 & 504 & 604 & 56.00 & 46,46 & 4 & 3 & 3.12 & 2.72 & 26 & 15 \\
\hline B1T2 & 10 & 10 & 11 & 10 & 10 & 10 & 25 & 12 & 119 & 91 & 6.26 & 5.69 & 1348 & 1021 & 67.40 & 48,61 & 6 & 4 & 3.17 & 3.42 & 30 & 32 \\
\hline P1 & 10 & 10 & 10 & 10 & 10 & 10 & 16 & 14 & 105 & 92 & 6.56 & 5.75 & 1290 & 1190 & 64.50 & 59.50 & 6 & 5 & 3.08 & 2.58 & 26 & 25 \\
\hline $0 \pi 1$ & 10 & 9 & 10 & 9 & 10 & 10 & 15 & 12 & 104 & 85 & 6.50 & 5.67 & 740 & 927 & 49.33 & 51.68 & 4 & 4 & 3.37 & 2.85 & 25 & 25 \\
\hline Con2 & 9 & 10 & 9 & 10 & 9 & 10 & 12 & 12 & 107 & 106 & 5.63 & 5.89 & 1300 & 1102 & 65.00 & 50.09 & 5 & 6 & 3.73 & 3.35 & 26 & 29 \\
\hline On 3 & 9 & 8 & 9 & B & 9 & 9 & 11 & 10 & 69 & 60 & 5.31 & 5.45 & 889 & 277 & 55.56 & 30.77 & 3 & 2 & 3.42 & 2.72 & 28 & 18 \\
\hline OnH1 & 9 & 9 & 11 & 9 & 9 & 9 & 14 & 14 & 146 & 109 & 5.41 & 5.45 & 1301 & 873 & 59.13 & 54.56 & 8 & 5 & 3.69 & 2.87 & 40 & 29 \\
\hline By 1 & 10 & 10 & 12 & 11 & 10 & 10 & 16 & 15 & 163 & 117 & 7.08 & 6.50 & 1392 & 1510 & 58.00 & 68.63 & 9 & 8 & 4.52 & 3.63 & 41 & 24 \\
\hline By 2 & 9 & 9 & 10 & 9 & 9 & 9 & 14 & 12 & 108 & 120 & 5.68 & 6.00 & 1133 & 1262 & 49.26 & 52.58 & 5 & 5 & 3.33 & 3.79 & 29 & 33 \\
\hline By3 & 10 & 10 & 11 & 10 & 10 & 10 & 14 & 13 & 125 & 100 & 5.95 & 5.88 & 1234 & 1218 & 56.09 & 60.90 & 4 & 4 & 2.68 & 3.59 & 30 & 23 \\
\hline By 4 & 8 & - & 8 & - & 7 & - & 8 & - & 48 & - & 5.33 & - & 826 & $=$ & 48.58 & - & 2 & - & 2.93 & - & 19 & $=$ \\
\hline By 5 & B & 7 & 8 & 7 & 8 & 7 & 11 & 9 & 75 & 32 & 5.35 & 5.00 & 650 & 432 & 65.00 & 43.20 & 3 & 2 & 2.98 & 3.08 & 19 & 20 \\
\hline 21 & 9 & - & 9 & $=$ & 9 & - & 13 & - & 107 & - & 5.94 & - & 725 & - & 60.41 & - & 6 & - & 1.98 & - & 17 & - \\
\hline 22 & 0 & 0 & 0 & 8 & 0 & 0 & 0 & 0 & 0 & 0 & 0.00 & 0.00 & 0 & 0 & 0.00 & 0.00 & 0 & 0 & 0.00 & 0.00 & 0 & 0 \\
\hline 23 & 2 & 1 & 1 & 3 & 2 & 2 & 3 & 2 & 6 & 3 & 2.00 & 3.00 & 73 & 7 & 24.33 & 7.00 & 0 & 0 & 2.18 & 0.00 & 6 & 1 \\
\hline 24 & 8 & 9 & 9 & 8 & 8 & 11 & 8 & 8 & 62 & 93 & 4.43 & 5.27 & 670 & 919 & 47.85 & 48.36 & 3 & 4 & 3.23 & 0.42 & 19 & 32 \\
\hline $\mathrm{z} 5$ & 4 & 3 & 3 & 2 & 4 & 2 & 4 & 3 & 12 & 9 & 3.00 & 2.25 & 62 & 62 & 12.40 & 12.40 & 0 & 0 & 2.09 & 2.19 & 11 & 7 \\
\hline 26 & 5 & 4 & 4 & 5 & 5 & 4 & 6 & 9 & 30 & 29 & 3.00 & 3.22 & 201 & 136 & 16.75 & 27.20 & 1 & 1 & 2.99 & 2.46 & 15 & 11 \\
\hline 27 & 8 & 6 & 6 & 5 & 8 & 6 & 7 & 9 & 39 & 29 & 3.54 & 3.62 & 489 & 367 & 44.45 & 36.70 & 2 & 1 & 3.07 & 2.59 & 28 & 18 \\
\hline zol & 9 & 8 & 8 & 9 & 9 & 10 & 9 & 12 & 93 & 77 & 6.64 & 6.42 & 1124 & 848 & 62.44 & 70.66 & 5 & 3 & 3.58 & 2.82 & 28 & 16 \\
\hline zuz & 8 & 9 & 9 & 9 & 8 & 11 & $B$ & 13 & 80 & 88 & 5.71 & 5.87 & 860 & 1131 & 66.15 & 66.52 & 4 & 5 & 2.49 & 3.30 & 23 & 25 \\
\hline 2B1 & 6 & 10 & 10 & 10 & 6 & 10 & 7 & 9 & 22 & 81 & 4.40 & 6.23 & 368 & 844 & 40.88 & 64.92 & 0 & 4 & 3.39 & 3.93 & 17 & 25 \\
\hline 221 & 9 & 9 & 9 & 10 & 9 & 11 & 9 & 14 & 76 & $9 B$ & 5.06 & 5.44 & 1361 & 1293 & 54.44 & 5 B. 77 & 4 & 4 & 3.39 & 3.48 & 30 & 32 \\
\hline ZAI & 10 & 10 & 10 & 10 & 11 & 14 & 10 & 15 & 99 & 93 & 6.60 & 6.20 & 1896 & 1351 & 72.90 & 67.55 & 7 & 5 & 3.15 & 3.16 & 35 & 25 \\
\hline $2 A 2$ & 9 & 8 & 8 & 8 & 9 & 12 & 9 & 13 & 89 & 83 & 5.56 & 5.53 & 1407 & 1010 & 63.95 & 56.11 & 6 & 2 & 2.95 & 3.23 & 34 & 22 \\
\hline $2 A 3$ & 9 & 8 & 8 & $B$ & 9 & 12 & 9 & 11 & 49 & 61 & 4.08 & 5.08 & 979 & 611 & 54.38 & 47.00 & 2 & 3 & 2.18 & 2.54 & 27 & 21 \\
\hline In] & 8 & 3 & 3 & 4 & 8 & 2 & 8 & 9 & 68 & 11 & 4.00 & 2.75 & 736 & 115 & 49.06 & 23.00 & 3 & 0 & 2.62 & 1.90 & 25 & 14 \\
\hline $\operatorname{In} 2$ & 7 & 8 & 8 & 9 & 7 & 9 & 7 & 11 & 39 & 52 & 4.87 & 4.33 & 852 & 700 & 56.80 & 50.00 & 2 & 4 & 2.84 & 2.46 & 21 & 23 \\
\hline Eg1 & 10 & - & 10 & $=$ & 10 & $=$ & 12 & - & 70 & - & 5.00 & - & 570 & - & 43.60 & - & 4 & - & 3.58 & - & 22 & - \\
\hline Eg2 & 8 & 6 & 8 & 6 & 6 & 5 & $B$ & 9 & 40 & 50 & 3.63 & 3.85 & 361 & 423 & 36.10 & 42.30 & 1 & 2 & 3.40 & 0.58 & 22 & 12 \\
\hline $\mathrm{Eg} 3$ & 9 & 5 & 11 & 5 & 9 & 5 & 13 & 4 & 121 & 32 & 5.04 & 3.55 & 1659 & 223 & 57.20 & 31.85 & 6 & 1 & 3.82 & 2.92 & 42 & 18 \\
\hline EgB1 & 10 & 10 & 10 & 12 & 10 & 10 & 14 & 16 & 82 & 138 & 5.85 & 6.27 & 1604 & 1820 & 61.69 & 56.87 & 5 & 8 & 2.55 & 3.97 & 33 & 38 \\
\hline $\mathrm{EgB2}$ & 9 & 9 & 9 & 9 & 9 & 9 & 14 & 14 & 93 & 81 & 5.47 & 5.78 & 1591 & 1184 & 63.64 & 65.77 & 5 & 5 & 2.80 & 1.89 & 27 & 22 \\
\hline
\end{tabular}


Tableau 3. Classes de qualité, rangs des valeurs du BMWP' (A : d'après Alba-Tercedor \& Sánchez-Ortega 1988, B : d'après les données de la présente étude) et couleurs à utiliser dans les représentatjons cartographiques.

Table 3. Quality classes, range of BMWP' values (A : according to Alba-Tercedor \& Sánchez-Ortega 1988, B : according to grouping with data of study) and colours to use in cartographic representations.

\begin{tabular}{lccll}
\hline Class & $\begin{array}{l}\text { Values of BMWP } \\
\text { A }\end{array}$ & B & Significance & Colour \\
\hline I & $>150$ & $>135$ & Very clean waters & Blue \\
I & $101-120$ & $95-135$ & Clean waters & Blue \\
II & $61-100$ & $65-94$ & Waters with some disturbance & Green \\
III & $36-60$ & $45-64$ & Polluted waters & Yellow \\
IV & $16-35$ & $20-44$ & Seriously polluted waters & Orange \\
V & $<15$ & $<20$ & Grossly polluted waters & Red \\
\hline
\end{tabular}

Also it would be necessary to extend the list of taxons proposed by Alba-Tercedor \& SánchezOrtega (1988) including other iberian groups, and to modify the scores of water quality depending on the peninsular area where the samples came from.

\section{Acknowledgements}

This study is supported by the Viceconsejeria de Medio Ambiente of the Basque Country Government as part of the «Caracterización hidrobiológica de las redes fluviales de Alava y Guipúzcoa ». We would like to thank Dr. Javier Alba-Tercedor (Universidad de Granada), Dr. Javier Garcia-Avilés (Universidad Autónoma de Madrid) and Dr. Gloria González (LIMNOS, S.A., Barcelona) for data contribution and personal commentaries.

\section{References}

Alba-Tercedor J. \& Sánchez-Ortega A. 1988. - Un método rápido y simple para evaluar la calidad biológica de las aguas corrientes basado en el de Hellawell (1978). Limnélica, 4 : 51-56.

Armitage P.D., Moss D., Wright J.F. \& Furse M.T. 1983, The performance of a new biological water quality score system based on macroinvertebrates over a wide range of unpolluted running-water sites. Wat. Res., 17 : 333-347.

Balloch D., Davies C.E. \& Jones F.H. 1976. - Biological assessment of water quality in three rivers : the North Esk (Scotland), the lvel (England) and the Taf (Wales). Wat. Pollut. Contr., $75: 92-110$.

Chandler J.R. 1970. - A biological approach to water quality management. Wat. Pollut. Contr., 69 : 415-422.

Domezain A., Guisasola I. \& Alba-Tercedor J. 1987. - Estudio de la incidencia de una piscifactoria en las comurtidades de macroinvertebrados acuáticos. Limnética. 3; 15 1-157.

Fjerdingstad E. 1964. - Pollution of streams estimated by benthal phytomicroorganisms. 1. A saprobic system based on communities of organisms and ecological factors. Int. Revue. ges. Hydrobiol., $49: 63 \cdot 131$.

Ghetti P.F., Bernini F., Bonazzi G., Cunsolo A. \& Ravanetti U. 1983. - Mappagio biologico di qualitả dei corsi d’acqua della provincia di Piacenza. Administ. Prov. Piacenza, CNR. « Promozione della qualità dell'ambiente-Progetto di trasferimento $»: 28 \mathbf{p}(+1$ map).
Ghetti P.F. \& Bonazzi G. 1977. - A comparison between various criteria for the interpretation of biological data in the analysis of the quality of running waters. Wat. Res., $11: 819-831$.

Kolkwitz R. \& Marsson M. 1902. - Grundsätze für dic biologische Beurteilung des Wassers nach seiner Flora und Fauna. Mitt. a. d. Kgl. Prüfungsanst f. Wasserversorg u. Abwässerbeseitigung zu Berlin, 1 : 33-72.

Kolkwitz R. \& Marsson M. 1908. - Ókologie der pflanzlichen Saprobien. Ber. Dr. Botam. Ges., 261 : 505-519.

Kolkwitz R. \& Marsson M. 1909. - Ökologie der tierischen Saprobien. Imt. Revue ges. Hydrobiol, 2 : 125-152.

Lang C., l'Eplattenier G, \& Reymond O. 1989. -- Water quality in rivers of western Switzerland : Application of an adaptable index based on benthic invertebrates. Aquat. Sc., 51 (3) : 224-234

Liebmann H. 1951. - Handbuch der Frischwasser-und Abwasserbiologie. Bd. I, 1. Aufl. Verlag Oldenbourg, Munich : $588 \mathrm{p}$.

Liebmann H, 1962. - Handbuch der Frischwasser-und Abwasserbiologie, Bd. Il, 2. Aufl. Oldenbourg, Munich. Verlag G. Fischer, Jena : I $160 \mathrm{p}$.

Mesanza J.M., Bargas D. \& Orive E. 1988. - Calidad del agua de los ríos de Bizkaia en base al uso de varios índices bióticos. Actas Congr. Biol. Amb., II Congr. Mund. Vasco, 2 : 181.195.

National Water Council 1981. - River quality: The 1980 survey and further outloolk. N.W.C., London.

Rodriguez P. \& Wright J.F. 1988. - Biological evaluation of the quality of three basque water courses. Actas Congr. Biol. Amb., Il Congr. Mund. Vasco, $2: 223-243$.

Rodriguez P. \& Wright J.F. 199I. - Description and evaluation of a sampling strategy for macroinvertebrate communities in Basque rivers (Spain). Hydrobiologia, 213: 113-124.

Sladecek V. 1961. - Zur biologischen Gliederung der hoheren Saprobitätsstufen. Arch. Hydrobiol, 58 : 103-121.

Sladecek V. 1967. - The ecological and physiological trends in the Saprobiology. Hydrobiologia, $30: 513-526$.

Sladecek V. 1973. - Systems of water quality from the biological point of view. Arch. Hydrobiol., $7: 1.218$.

Sneath P.H.A. \& Sokal R.R. 1973. - Numerical taxonomy, Freeman \& Co., San Francisco : 573 p.

Sokal R.R. \& Rohlf F.J. 1969. - Biometry, the principles and practice of statistics in biological research. Freeman \& Co., San Francisco : 776. p. 
Tolkamp H.H. 1985, - Using several indices for biological assessment of water quality in running water. Verh. internat. Ver. Limnol, 22 : 2281-2286.

Verneaux J. 1982. - Réflexions sur l'appréciation de la qualité des eaux courantes à l'aide de méthodes biologiques. $A$ nn. Sci. Univ. Besançon, Biol. Anim., 4ème Sér., 3 : 3-9.

Verneaux J., Galmiche P., Janier F. \& Monnot A. 1982. - Une nouvelle mét hode pratique d'évaluation de la qualité des eaux courantes. Un indice biologique de qualité générale (I.B.G.). Ann. Sci. Univ. Besançon, Biol. Anim., 4ème sér., 3 :11-21.

Verneaux J. \& Tuffery G. 1967. - Une méthode zoologique pratique de détermination de la qualité biologique des eaux courantes. Indices biotiques. Ann. Sci, Univ. Besançon, Zool, $3: 79-90$.
Washington H.G. 1984. - Diversity and similarity indices. A review with special relevance to aquatic ecosystems. Wat. Res., $18: 653-694$.

Woodiwiss F.S. 1964. - The biological system of stream classification used by the Trent River Board. Chem. Indust., 14 : 443-447.

Woodiwiss F.S. 1978. - Biological water assessment methods. Severn-Trent Authorities, U.K. 